Note

\title{
Effects of Soluble and Insoluble Fiber Preparations Isolated from Oat, Barley, and Wheat on Liver Cholesterol Accumulation in Cholesterol-Fed Rats
}

\author{
Taishi Oda, ${ }^{1,2}$ Seiichiro Aoe, ${ }^{1}$ Hiroo Sanada, ${ }^{2}$ \\ and Yūkō AYANO ${ }^{2}$ \\ ${ }^{1}$ Technical Research Institute, Snow Brand Milk Products, \\ Kawagoe, Saitama 350, Japan \\ ${ }^{2}$ Department of Bioproduction Science, Faculty of Horticulture, \\ Chiba University, Matsudo, Chiba 271, Japan
}

(Received September 14, 1992)

\begin{abstract}
Summary Effects of soluble and insoluble fiber preprations isolated from defatted oat, barley, and wheat on liver cholesterol accumulation were examined in cholesterol-fed rats. The soluble and insoluble fiber preparations were isolated by a modification of the procedure of Asp et al. Male Sprague-Dawley rats, aged 5 weeks, were fed a control diet containing $5 \%$ cellulose or diets containing soluble and insoluble fiber preparations for 9 days. Soluble fiber preparations from oat, barley, and wheat were added to diets corresponding to $1.9,2.8$, and $0.6 \%$ fiber, respectively. Insoluble fiber preparations from oat, barley, and wheat were added to diets corresponding to 3.1, 2.2, and 4.4\% fiber, respectively. All of the soluble fiber preparations suppressed liver cholesterol accumulation, but they did not suppress the elevation of plasma cholesterol concentrations. A significant inverse relationship between the quantity of ingested soluble fiber and liver cholesterol accumulation was observed; $n=28$, $r=-0.943, p<0.0001$. None of the insoluble fiber preparations had any significant effect on liver and plasma cholesterol concentrations. These results indicate that the water-soluble fractions of oat, barley, and wheat are the active components that suppressed liver cholesterol accumulation, and that these effects are related to the quantity of ingested soluble fiber.
\end{abstract}

Key Words dietary fiber, oat, barley, wheat, cholesterol-lowering effect, liver cholesterol, rats

Oat, barley, and wheat are important sources of dietary fiber; oat and barley are rich in water-soluble fiber but wheat is rich in water-insoluble fiber $(1,2)$. Soluble fibers of oat and barley are mainly composed of $\beta$-glucan (3), whereas soluble fiber of wheat contains pentosan and $\beta$-glucan $(4,5)$.

Oat and barley lower plasma and liver cholesterol concentrations in experimental animals (6-10). Shinnick et al. (7) reported that diet supplemented with $10 \%$ 
fiber from high fiber oat prevented the elevation of plasma and liver cholesterol concentrations in rats, but $5 \%$ fiber from oat prevented only the elevation of liver cholesterol concentrations. Wheat has no cholesterol-lowering effect, because most of its dietary fiber is insoluble (11). However, we previously showed that diets with $5 \%$ fiber from defatted oat, barley, and wheat suppressed liver cholesterol accumulation in cholesterol-fed rats (12). We also presented that the suppressive effects of defatted oat, barley, and wheat on liver cholesterol accumulation were related to the quantity of ingested soluble fiber from these cereal flours (12). On the other hand, several workers (13-17) found that soluble-fiber-enriched fractions of oat, barley, and wheat lowered plasma and liver cholesterol concentrations in rats. Therefore, the cholesterol-lowering effects of oat, barley, and wheat might be attributed to soluble fiber consumed. Nevertheless, processed oat hull, which is $>95 \%$ insoluble fiber, has an effect on the lowering of plasma and liver cholesterol concentrations on certain conditions (18). The amino acid content of oat may also promote a hypocholesterolemic response (19).

The objective of this research was to specify the active fractions of oat, barley, and wheat that suppressed liver cholesterol accumulation in rats. This study examined the effects of soluble and insoluble fiber preparations isolated from defatted oat, barley, and wheat on liver cholesterol accumulation in cholesterol-fed rats.

Materials and methods. 1) Samples: Cereals were obtained from the following: dehulled oat from Quaker Oats Co., polished barley (extraction rate $73 \%$ ) from Abe Polish Co., and whole grain wheat flour from Nissin Flour Milling Co. These cereal flours were defatted with $n$-hexane. Oat and barley flours were mixed with $n$-hexane in 10 volumes, and the mixtures were stirred and centrifuged. Wheat flour was mixed with $n$-hexane in 3 volumes, and the mixture was stirred and filtered (Milk filter, Azumi Filter Paper Co.). The residue from the initial removal of fat was defatted two times under the same conditions as the first. These residues were air-dried at $60^{\circ} \mathrm{C}$, milled, and sieved through a $0.59 \mathrm{~mm}$ screen. Removal of fat from oat, barley, and wheat flours reduced their fat content by 82 , 79 , and $60 \%$, respectively.

Soluble and insoluble fiber preparations were isolated by a modification of the procedure of Asp et al. (20). Defatted cereal flour was mixed with $0.2 \mathrm{~m}$ sodium phosphate buffer solution, and the mixture was heated with Termamyl 120L (Novo Industry Co.), and cooled. The protein and starch in the mixture was digested with Pepsin NF (Merck \& Co.) and Pancreatin $4 \times$ U.S.P. (Sigma Chemical Co.), respectively, and then the enzymes were inactivated at $80^{\circ} \mathrm{C}$ for $10 \mathrm{~min}$. The insoluble residue was isolated by centrifugation, and then washed with water, freeze-dried, milled, and sieved through a $0.59 \mathrm{~mm}$ screen. The combined water extract was dialyzed against tap water and poured into four volumes of ethanol. The precipitate was collected by centrifugation, redissolved in water, freeze-dried, milled, and sieved through a $0.59 \mathrm{~mm}$ screen. White or brown powders were obtained.

2) Analyses: The dietary fiber content in soluble and insoluble fiber 
preparations was analyzed by the method of Prosky et al.(1). The dietary fiber residue isolated from soluble fiber preparations by the previous method $(I)$ was hydrolyzed to alditol acetates according to the procedure of Theander and Åman (21). The alditol acetates formed were quantified on a Hewlett Packard model 5890 gas chromatograph fitted with a fused silica DB-1 column (diameter, $0.53 \mathrm{~mm}$; length, $30 \mathrm{~m}$; nitrogen gas flow, $10 \mathrm{ml} / \mathrm{min} ; 190^{\circ} \mathrm{C}$ ). Quantitative estimates of neutral sugars presented were made by comparing their peak areas with that of 2-deoxy-D-glucose, as an internal standard. Uronic acid content was determined by the carbazole method of Bitter and Muir (22). Liver cholesterol was extracted with chloroform-methanol $(2: 1, \mathrm{v} / \mathrm{v})$ by the method of Folch et al. (23), and redissolved in isopropanol. Liver and plasma cholesterol concentrations were determined by the enzymatic procedure (Determiner TC5, Kyowa Medex Co.). Apparent accumulation ratio of liver cholesterol to ingested cholesterol was calculated according to Tsuji and Nakagawa (24).

3) Animals and diets: Male Sprague-Dawley rats, aged 4 weeks, were purchased from Clea Japan (Tokyo). Animals were housed in individual stainless steel cages and fed a cholesterol- and sodium-cholate-free semipurified diet for 4 days. Diets and tap water were provided ad libitum. The rats were then fed a hypercholesterolemic diet containing 5\% cellulose (control) or diets containing soluble and insoluble fiber preparations for 9 days. All diets contained the following $(\%, \mathrm{w} / \mathrm{w})$ : sucrose, 20; lard, 9; corn oil, 1; AIN-76 mineral mixture (Oriental Yeast Co.), 3.5; AIN-76 vitamin mixture (Oriental Yeast Co.), 1; cholesterol, 1; sodium cholate, 0.25 ; choline chloride, 0.15 . Casein as a protein source was added to adjust nitrogen at $3 \%$ in all diets. The previous report (12) showed that the amounts of soluble fiber from oat, barley, and wheat in diets were $1.9,2.8$, and $0.6 \%$, respectively, when these cereal flours were added to diets corresponding to $5 \%$ dietary fiber. In the same manner, the amounts of insoluble fiber from oat, barley, and wheat in diets were $3.1,2.2$, and $4.4 \%$, respectively. Therefore, soluble fiber preparations from oat, barley, and wheat were added to diets corresponding to $1.9,2.8$, and $0.6 \%$ fiber, respectively. Insoluble fiber preparations from oat, barley, and wheat were added to diets corresponding to $3.1,2.2$, and $4.4 \%$ fiber, respectively. Cellulose powder was included to adjust fiber at $5 \%$ in diets containing soluble and insoluble fiber preparations. Corn starch was supplemented to adjust $100 \%$ in each diet. Ingested soluble fiber was calculated from food intake and content of soluble fiber in diet. Ingested insoluble fiber, except cellulose consumed, was calculated from food intake and content of insoluble fiber except cellulose in diet. After the 9-day feeding period, the rats were fasted for $17 \mathrm{~h}$ and sacrificed, and liver was weighed. Livers were freeze-dried, milled, and kept at $-20^{\circ} \mathrm{C}$ until analysis of cholesterol. Blood was withdrawn from postcava under ether anesthesia, and collected in a heparinized test tube. The blood was centrifuged, and plasma was collected for analysis of cholesterol.

Statistical analysis was made by analysis of variance and Tukey's multiple range test (25). Linear regression analysis was performed on some of the data, and Vol. 39, No. 1, 1993 
Table 1. Components of soluble and insoluble fiber preparations isolated from defatted oat, barley, and wheat $(\%)$.

\begin{tabular}{|c|c|c|c|c|c|c|}
\hline & \multicolumn{3}{|c|}{ Soluble fiber preparations } & \multicolumn{3}{|c|}{ Insoluble fiber preparations } \\
\hline & Oat & Barley & Wheat & Oat & Barley & Wheat \\
\hline Moisture & 7.4 & 6.9 & 5.5 & 3.1 & 3.0 & 3.9 \\
\hline Crude protein ${ }^{1}$ & 16.6 & 11.9 & 19.3 & 24.3 & 7.9 & 11.1 \\
\hline Ash & 16.3 & 6.4 & 21.3 & 2.5 & 1.8 & 1.8 \\
\hline Dietary fiber ${ }^{2}$ & 51.1 & 68.1 & 49.5 & 56.5 & 82.1 & 80.3 \\
\hline Carbohydrate $^{3}$ & 8.6 & 6.7 & 4.4 & 13.6 & 5.2 & 2.9 \\
\hline
\end{tabular}

${ }^{1}$ Calculated by $\mathrm{N} \times 6.25 . \quad{ }^{2}$ Measured by the method of Prosky et al. (1). ${ }^{3}$ Calculated as the difference between total and the sum of moisture, crude protein, ash, and dietary fiber.

Table 2. Percent distribution of undigested carbohydrate ${ }^{1}$ in soluble fiber preparations from oat, barley, and wheat (\%).

\begin{tabular}{lrcc}
\hline & \multicolumn{3}{c}{ Soluble fiber preparations } \\
\cline { 2 - 4 } & Oat & Barley & Wheat \\
\hline Arabinose $^{1}$ & 4.9 & 7.8 & 31.7 \\
Xylose $^{2}$ & 2.9 & 10.6 & 39.1 \\
Glucose $^{2}$ & 77.4 & 73.6 & 10.7 \\
Galactose $^{2}$ & 4.8 & 1.9 & 10.6 \\
Mannose $^{2}$ & 4.8 & 3.7 & 3.4 \\
Uronic acid $^{3}$ & 5.2 & 2.4 & 4.5 \\
\hline
\end{tabular}

${ }^{1}$ Isolated by the method of Prosky et al. (1). ${ }^{2}$ Measured by the method of Theander and Åman (21). ${ }^{3}$ Measured by the method of Bitter and Muir (22).

correlation coefficient was determined (25).

Results and discussion. Components of soluble and insoluble fiber preparations are shown in Table 1. Dietary fiber content in the insoluble fiber preparation isolated from barley was the highest among six fiber preparations, and that in the soluble fiber preparation from wheat was the lowest. Percent distribution of undigested carbohydrate in the soluble fiber preparations is presented in Table 2. Undigested carbohydrate in the soluble fiber preparations from oat and barley was mainly composed of glucose, whereas that from wheat was mainly arabinose and xylose.

The effects of soluble and insoluble fiber preparations from oat, barley, and wheat on liver and plasma cholesterol concentrations in cholestrol-fed rats are shown in Table 3 . There were no differences in body weight gain, food intake, or 


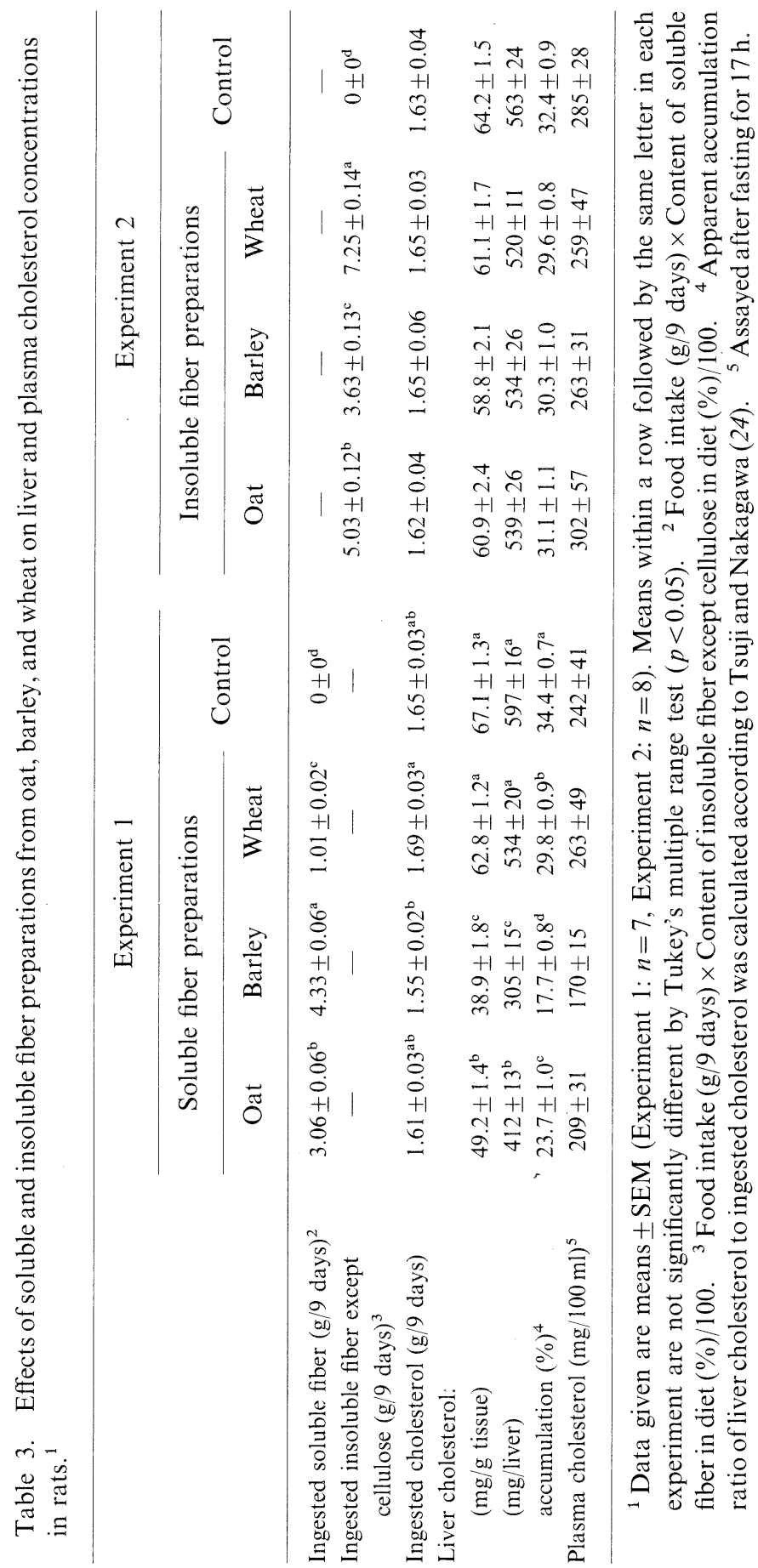

Vol. 39, No. 1, 1993 
food efficiency in rats fed diets containing soluble and insoluble fiber preparations, comparing with the control group (data not presented). Soluble fiber preparation from barley decreased liver weight (data not presented). All of the soluble fiber preparations suppressed liver cholesterol accumulation, consistent with previous studies $(6,13-17)$, whereas they did not suppress the elevation of plasma cholesterol concentrations. Several investigators, however, found that soluble-fiber-enriched fractions of oat, barley, and wheat lowered plasma cholesterol concentrations in cholesterol-fed rats $(13-16)$. Shinnick et al. (7) reported that adding high fiber oat flour to hypercholesterolemic diet reduced the magnitude of the elevation of plasma cholesterol concentrations in rats. In this study, the amount of soluble fiber provided by each diet might have insufficient to significant decrease plasma cholesterol concentrations. Linear regression analysis was performed on the quantity of ingested soluble fiber and liver cholesterol accumulation. Statistical analysis revealed the highly significant regression line $(n=28, r=-0.943, p<0.0001)$. On the other hand, none of the insoluble fiber preparations had any significant effect on liver and plasma cholesterol concentrations (Table 3). No significant relationship between the quantity of ingested insoluble fiber except cellulose and liver cholesterol accumulation was observed (data not presented). These results indicated that the water-soluble fractions of oat, barley, and wheat are the active components that suppressed liver cholesterol accmulation in rats. Other fractions except soluble fiber, such as protein, may have less liver-cholesterol-lowering activity, because the suppressive effects of defatted oat, barley, and wheat, and soluble fiber preparations from these cereals on liver cholesterol accumulation were related to the quantity of ingested soluble fiber only (12).

\section{REFERENCES}

1) Prosky, L., Asp, N.-G., Schweizer, T. F., DeVries, J. W., and Furda, I. (1988): Determination of insoluble, soluble, and total dietary fiber in foods and food products. J. Assoc. Off. Anal. Chem., 71, 1017-1023.

2) Nyman, M., Siljeström, M., Pedersen, B., Bach-Knudsen, K. E., Asp, N.-G., Johansson, C.-G., and Eggum, B. O. (1984): Dietary fiber content and composition in six cereals at different extraction rates. Cereal Chem., 61, 14-19.

3) Wood, P. J., Paton, D., and Siddiqui, I. R. (1976): Determination of $\beta$-glucan in oats and barley. Cereal Chem., 54, 524-533.

4) Pomeranz, Y. (1988): Composition and functionality of wheat flour components, in Wheat: Chemistry and Technology Vol. 2, ed. by Pomeranz, Y., Am. Assoc. Cereal Chem., St. Paul, pp. 219-370.

5) Lineback, D. K., and Rasper, V. F. (1988): Wheat carbohydrates, in Wheat: Chemistry and Technology Vol. 1, ed. by Pomeranz, Y., Am. Assoc. Cereal Chem., St. Paul, pp. 277-372.

6) Anderson, J. W., and Chen, W. L. (1986): Cholesterol-lowering properties of oat products, in Oats: Chemistry and Technology, ed. by Webster, F. H., Am. Assoc. Cereal Chem., St. Paul, pp. 309-333.

7) Shinnick, F. L., Ink, S. L., and Marlett, J. A. (1990): Dose response to a dietary oat 
bran fraction in cholesterol-fed rats. J. Nutr., 120, 561-568.

8) Takase, S., Kurihara, N., and Michi, K. (1971): Effect of cereals, especially barley on plasma cholesterol content. Eiyo to Shokuryo (J. Jpn. Soc. Nutr. Food Sci.) 23, 426-429.

9) Fadel, J. G., Newman, R. K., Newman, C. W., and Barnes, A. E. (1987): Hypocholesterolemic effect of $\beta$-glucans in different barley diets fed to broiler chicks. Nutr. Rep. Int., 35, 1049-1058.

10) Martinez, V. M., Newman, R. K., and Newman, C. W. (1992): Barley diets with different fat sources have hypocholesterolemic effects in chicks. J. Nutr., 122, 1070-1076.

11) Betschart, A. A. (1988): Nutritional quality of wheat and wheat foods, in Wheat: Chemistry and Technology Vol. 2, ed. by Pomeranz, Y., Am. Assoc. Cereal Chem., St. Paul, pp. 91-130.

12) Oda, T., Aoe, S., Sanada, H., and Ayano, Y. (1992): Effects of oat, barley and wheat on liver and plasma cholesterol concentrations in cholesterol-fed rats. Nippon Eiyo Shokuryo Gakkaishi (J. Jpn. Soc. Nutr. Food Sci.), 45, 560-563.

13) Chen, W. L., Anderson, J. W., and Gould, M. R. (1981): Effects of oat bran, oat gum and pectin on lipid metabolism of cholesterol-fed rats. Nutr. Rep. Int., 24, 1093-1097.

14) Klopfenstein, C. F., and Hoseney, R. C. (1987): Cholesterol-lowering effect of $\beta$-glucanenriched bread. Nutr. Rep. Int., 36, 1091-1098.

15) Wood, P. J., Anderson, J. W., Braaten, J. T., Cave, N. A., Scott, F. W., and Vachon, C. (1989): Physiological effects of $\beta$-D-glucan rich fractions from oats. Cereal Foods World, 34, 878-882.

16) Jennings, C. D., Boleyn, K., Bridges, S. R., Wood, P. J., and Anderson, J. W. (1988): A comparison of the lipid-lowering and intestinal morphological effects of cholestyramine, chitosan, and oat gum in rats. Proc. Soc. Exp. Biol. Med., 189, 13-20.

17) Oda, T., Aoe, S., Sanada, H., and Ayano, Y. (1991): Effect of ingested oat and barley gums on plasma and liver lipid concentrations in genetically obese and lean Zucker rats. Nippon Eiyo Shokuryo Gakkaishi (J. Jpn. Soc. Nutr. Food Sci.), 44, 455-460.

18) López-Guisa, J. M., Harned, M. C., Dubielzig, R., Rao, S. C., and Marlett, J. A. (1988): Processed oat hulls as potential dietary fiber sources in rats. J. Nutr., 118, 953-962.

19) Kritchevsky, D., Tepper, S. A., and Klurfeld, D. M. (1987): Dietary protein and atherosclerosis. J. Am. Oil Chem. Soc., 64, 1167-1171.

20) Asp, N.-G., Johansson, C. G., Hallmer, H., and Siljeström, M. (1983): Rapid enzymatic assay of insoluble and soluble dietary fiber. J. Agric. Food Chem., 31, 476-482.

21) Theander, O., and Åman, P. (1979): Studies of dietary fibres: Analysis and chemical characterization of water-soluble and water-insoluble dietary fibres. Swed. J. Agric. Res., 9, 97-106.

22) Bitter, T., and Muir, H. M. (1962): A modified uronic acid carbazole reaction. Anal. Biochem., 4, 330-334.

23) Folch, J., Lees, M., and Sloane-Stanley, G. H. (1957): A simple method for the isolation and purification of total lipids from animal tissues. J. Biol. Chem., 226, 497-509.

24) Tsuji, K., and Nakagawa, T. (1984): Effects of simultaneous feeding of konjac mannan and cellulose on the growth, organ weight and lipid levels in hypercholesterolemic rats. Nutr. Rep. Int., 30, 19-25.

25) SAS Institute SAS/STAT ${ }^{\mathrm{TM}}$ Guide for Personal Computers, Version 6 Ed. (1985), ed. by Joyner, S. P., SAS Institute, Cary, NC 